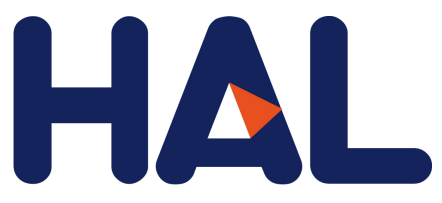

archives-ouvertes

\title{
Considering Sync Word and Header Error Rates for Performance Assessment in LoRa System
}

\author{
Vincent Savaux, Christophe Delacourt, Patrick Savelli
}

\section{To cite this version:}

Vincent Savaux, Christophe Delacourt, Patrick Savelli. Considering Sync Word and Header Error Rates for Performance Assessment in LoRa System. Wireless telecommunications Symposium, Apr 2021, San Francisco, France. hal-03200449

\section{HAL Id: hal-03200449 \\ https://hal.archives-ouvertes.fr/hal-03200449}

Submitted on 16 Apr 2021

HAL is a multi-disciplinary open access archive for the deposit and dissemination of scientific research documents, whether they are published or not. The documents may come from teaching and research institutions in France or abroad, or from public or private research centers.
L'archive ouverte pluridisciplinaire HAL, est destinée au dépôt et à la diffusion de documents scientifiques de niveau recherche, publiés ou non, émanant des établissements d'enseignement et de recherche français ou étrangers, des laboratoires publics ou privés. 


\title{
Considering Sync Word and Header Error Rates for Performance Assessment in LoRa System
}

\author{
Vincent Savaux, Christophe Delacourt, and Patrick Savelli \\ $\mathrm{b}<>$ com, Rennes, France \\ Email: vincent.savaux,christophe.delacourt,patrick.savelli@b-com.com
}

\begin{abstract}
This paper deals with a general and complete analysis of the frame error rate in LoRa system, not only considering the payload error rate, but also the sync word (carrying network information) and the header (carrying control information) error rates. It is proved that, in the high signal-to-noise ratio (SNR) range, it is more likely to reject a LoRa frame due to an erroneous sync word estimation rather than an erroneous header or payload decoding, due to the fact that it is not coded. The theoretical results are then verified through simulations.

Index Terms-LoRa, Error Rate, Channel.
\end{abstract}

\section{INTRODUCTION}

The last few years have seen the large scale deployment of Internet of things (IoT) networks. Among the different emerging technologies, LoRa addresses the low power wide area network (LPWAN) segment. LPWAN relies on low cost, long battery life devices, able to achieve long range data communication with low data rates. These requirements typically target remote sensing for smart cities, smart agriculture or industrial applications to name a few. The LoRa technology is originally based on a proprietary physical layer (PHY) developed by Semtech corp., and the system has been promoted by the LoRa Alliance, that specifies the LoRaWAN o pen protocol.

The performance of the LoRa PHY in terms of bit error rate (BER) is being widely studied in the literature. In [1], [2], the LoRa receiver BER is analyzed in additive white Gaussian noise (AWGN) channel model. Thus, based on the results in [3], the authors of [1] suggest an analytical but complex BER expression as well as simpler approximations, while in [2] the BER is partially derived through a Monte-Carlo method. The theoretical BER performance of a LoRa signal receiver over more general channel models such as Rayleigh, Rice, and Nakagami is dealt with in [4], and approximations for large SNR values are provided in [5]. Alternatively in [6], the BER performance issue is tackled by considering interferers, and the effect of the channel coding is dealt with in [7] using both theoretical and experimental approaches.

However, all these performance analyses are carried out at the symbol or at the bit level, which does not reflect the fact that a whole LoRa payload within a LoRa frame is considered as erroneous as soon as one bit is erroneously decoded. This is the reason why the authors in [8] suggest a more thorough performance analysis of what they called frame error rate (FER), considering the whole payload where the binary stream is encoded (Hamming code) and interleaved. Despite

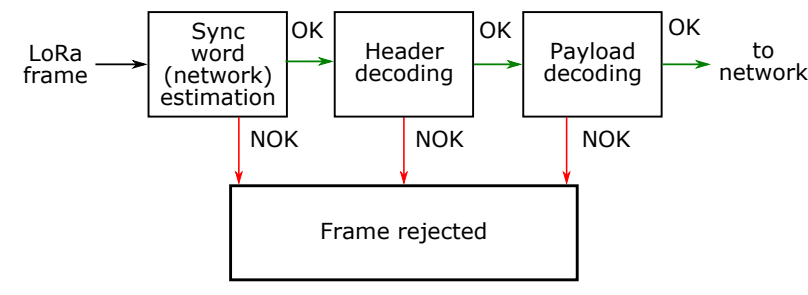

Fig. 1. Overview of LoRa decoding process: the sync word, the header, and the payload must be properly estimated/decoded otherwise the frame is rejected.

the quality and the rigor of their theoretical developments, we consider that the analysis in [8] leads to what we call the payload error rate (PLER) instead of the FER. In fact, such as depicted in Fig. 1, a LoRa frame is rejected, i.e. not transmitted to the network, not only if the payload is erroneously decoded, but also if the header (containing control information such as the payload size or the coding rate applied to the payload) is erroneously decoded, or if the sync word (two LoRa symbols within the preamble that carry the network information) is badly estimated. Thus, considering only the PLER limits the LoRa performance analysis to a theoretical use case, whereas in practice the FER analysis requires the sync word and the header error rates (SWER and HER respectively) besides the PLER.

In this paper, we extend the results in [8] to suggest a more general FER expression considering at the same time the contributions of the SWER, the HER and PLER. To this end, we also consider the more general Rice channel model [7] than the AWGN as in [8]. Moreover, an asymptotic performance analysis in the high SNR values region is also performed. The obtained theoretical results prove that above a given SNR value, the FER is dominated by the contribution of the sync word estimation errors. This is a interesting and counter-intuitive result as it shows that a LoRa frame may be rejected whereas the SNR would be sufficient for a proper payload decoding. The reason is that the sync word is not coded whereas the header and the payload are. Nevertheless, it is worth mentioning that the sync word is mandatory for the network deployment and then cannot be omitted in practical use cases. Thus, the FER considers the performance of the overall LoRa system whereas the BER is limited to a performance analysis considering the integrity of the data binary stream only. The theoretical results are then verified 
through simulations of LoRa signals in both AWGN and Rayleigh channels.

The rest of the paper is organized as follows: Section II presents the LoRa frame format and modulation scheme, as well as the demodulation process. It also provides the background related to the LoRa performance analysis. We develop the FER expression in Section III including the asymptotic analysis, and simulations results are shown in Section IV. Finally, we draw our conclusions in Section V.

Notations: $\mathbb{P}(A)$ means the probability of the event $A$, and $\mathcal{F}($.$) is the Fourier transform operation. The notations P_{b}, P_{s}$, $P_{s w}, P_{h} P_{p l}$, and $P_{f}$ indicate the binary, symbol, sync word, header, payload, and frame error probabilities, respectively.

\section{BACKGROUND ON LORA SYSTEM AND PERFORMANCE}

\section{A. LoRa Signal Model}

The LoRa modulation is based on chirp spread spectrum (CSS) featuring different spreading factors $(S F)$, allowing to control the bit rate and improve the range of the signal. The chirp symbol, originally described in [9], is a simple sine wave $x(t)=e^{j f_{c}(t) t}, t \in\left[0, T_{s}\right]$ where $T_{s}$ is a symbol period, and the instantaneous frequency $f_{c}(t)$ varies linearly with time within the range $\left[-\frac{B_{w}}{2}, \frac{B_{w}}{2}\right]$, where $B_{w}$ is called the frequency excursion of the chirp. The binary stream provided by the medium access control (MAC) layer is split by the PHY layer into sequences of $S F$ bits each, $S F \in\{7,8, . ., 12\}$, which are in turn Gray-mapped in one of the $M=2^{S F}$ possible chirp-based symbols. The modulation consists in a cyclic frequency shift of the base chirp $x(t)$. The way the LoRa symbols are built from the chirp $x(t)$ is described in numerous papers dealing with LoRa modulation [1], [4], [6], [9], and not further detailed in this paper as it is not mandatory for a good understanding of the hereby analysis.

A LoRa frame consists in three main parts as depicted on Fig. 2 and hereby described:

1) The preamble is used for detection of the LoRa frame and the time-frequency synchronization. Furthermore, it includes the sync word composed of two symbols, which carries the network identifier. The sync word is not coded.

2) The header contains the control information, such as the payload length and the channel coding rate applied to the payload. As described in [10], it is always composed of eight symbols, and the binary control information is coded with a Hamming code $(4,8)$, where the first and second integers denote the word length and the codeword length, respectively.

3) The payload carries data composed of $N_{p l}$ LoRa symbols, and the data is coded with Hamming code $\left(4, n_{H}\right)$ where $n_{H} \in\{5,6,7,8\}$.

In the following, we assume that the LoRa bandwidth is narrow enough to consider a one-tap block fading channel $h$. Then, if we denote by $x_{i}(t), i \in \mathbb{N}$, the $i$-th transmitted LoRa symbol within a frame, the received symbol $y_{i}(t)$, under the assumptions of perfect time-frequency synchronization, can be expressed as

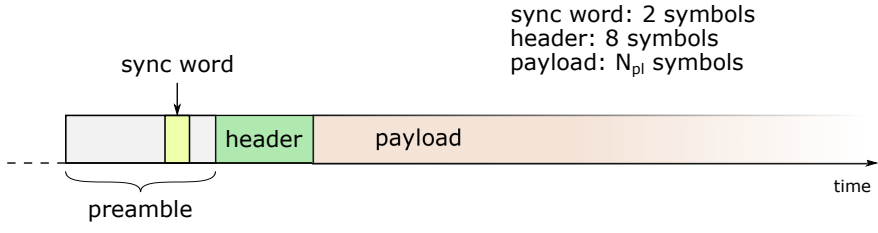

Fig. 2. A LoRa frame is composed of a preamble including the sync word (2 symbols), the header composed of eight symbols, and the payloads composed of $N_{p l}$ symbols.

$$
y_{i}(t)=h x_{i}(t)+w_{i}(t),
$$

where $w_{i}(t) \sim \mathcal{N}_{\mathbb{C}}\left(0, \sigma^{2}\right)$ is the complex AWGN. Furthermore, we suppose that $|h|$ obeys a Rice distribution, i.e. $h \sim \mathcal{N}_{\mathbb{C}}\left(\mu, \sigma_{h}^{2}\right)$, as it is a general model that includes other usual channel models, line-of-sight (LOS) (where $\mu \neq 0$ ) as well as non-LOS ones (where $\mu=0$ ). Since the chirp has a normalized module, the signal-to-noise ratio can be expressed as $S N R=\frac{\mathbb{E}\left\{|h|^{2}\right\}}{\sigma^{2}}$, which reduces to $S N R=\frac{1}{\sigma^{2}}$ in AWGN channel.

The demodulation and detection of the transmitted symbol from the observation (1) is deeply detailed in [1], [4], [6], [9]. The basic principle consists in multiplying the observation $y_{i}(t)$ by the conjugate of the base chirp, i.e. $x(t)^{*}$. Then, the frequency index corresponding to the maximum of the periodogram $\left|\mathcal{F}\left(y_{i}(t)\right) x(t)^{*}\right|^{2}$ yields the estimated transmitted symbol. If we denote by $m \in\{0,1, . ., M-1\}$ the actual transmitted symbol and by $\hat{m}$ its estimate, then the probability that the symbol is erroneously estimated is defined as $P_{s}=\mathbb{P}(\hat{m} \neq m)$. The remaining of the paper deals with the analysis of the error performance in the LoRa system.

\section{B. LoRa Performance}

Numerous papers deal with the performance of the LoRa system according to both BER [1], [2], [4], [6], [7] and payload error rate (PLER) [8]. The latter is especially relevant as it reflects that, in practice, the whole payload is considered as erroneous and therefore rejected (i.e. not forwarded to the network) if any constituting bit is badly decoded. It has been shown in [8] that a good approximation of the PLER, denoted by $P_{p l}$, can be expressed as

$$
P_{p l}=1-\left(\left(1-P_{b}\right)^{n_{H}}+n_{H} P_{b}\left(1-P_{b}\right)^{n_{H}-1}\right)^{\frac{N_{p l} S F}{n_{H}}},
$$

where $P_{b}$ is the bit error rate. In [8], the authors focus their analysis to the AWGN channel and use the approximated expression of $P_{b}$ such as suggested in [1]. We hereby extend the analysis to the more general Rice channel model. In fact, it has been proved in [4] that, in the absence of prior information, all the symbols can be considered as equiprobable so that the BER of the LoRa signal over a Rice channel, denoted by $P_{b}^{\text {Rice }}$, is expressed as 
TABLE I

OVERVIEW OF LORA BER ANALYSIS ACCORDING TO DIFFERENT MODELS AND APPROACHES.

\begin{tabular}{|l|l|l|}
\hline Reference & Channel & Approach \\
\hline$[1]-[3]$ & AWGN & $\begin{array}{l}\text { exact [3] } \\
\text { approximation [1] } \\
\text { Monte-Carlo [2] }\end{array}$ \\
\hline$[7]$ & AWGN+channel coding & approximation \\
\hline$[6]$ & AWGN+interference & approximation \\
\hline$[4]$ & Rayleigh, Rice, Nakagami & exact \\
\hline$[5]$ & Rayleigh, Rice & approximation \\
\hline
\end{tabular}

$$
\begin{aligned}
P_{b}^{\text {Rice }}= & -\frac{2^{S F-1}}{2^{S F}-1} \sum_{k=1}^{M-1} \frac{\left(\begin{array}{c}
M-1 \\
k
\end{array}\right)(-1)^{k} \sigma^{2}}{k M \sigma_{h}^{2}+(k+1) \sigma^{2}} \\
& \times \exp \left(-\frac{\lambda k M}{k N \sigma_{h}^{2}+(k+1) \sigma^{2}}\right),
\end{aligned}
$$

where $\lambda=|\mu|^{2}$ is called the non-central parameter. It must be noticed that the BER over Rayleigh and AWGN channels are straightforwardly deduced from (3) by setting $\lambda=0$ (Rayleigh) and $\left(\lambda=1, \sigma_{h}^{2}=0\right)$ (AWGN), respectively. However, it must be emphasized that (3) is not tractable, and that the computation of $\left(\begin{array}{c}M-1 \\ k\end{array}\right)$ may raise accuracy problems. This is the reason why approximations of the exact error probability (3) have been suggested for different channel models. Thus, Table I summarizes the approaches for LoRa BER analysis considering different channel models, which can be used for the following suggested FER expression.

However, although the PLER in (2) is a relevant performance indicator (note that a more sophisticated PLER approximation is also provided in [8]), it does account for all the possible LoRa frame rejection causes, as illustrated in Fig. 1. In fact, a LoRa frame is indeed rejected if the payload is erroneously decoded (highlighted by (2)), but also if the sync word is not properly estimated (which means the network is not recognized) or if the header is badly decoded (the control information required for the payload demodulation is erroneous). Thus, the complete FER expression including both SWER and HER in addition to PLER is hereby developed in Section III.

\section{FrAME ERROR RATE EXPRESSION}

In this section we derive the expressions of the sync word and header error rates, leading to the general FER.

\section{A. Sync Word Error Rate}

As aforementioned, the sync word is composed of two noncoded symbol. Thus, since the symbol error probability $P_{s}$ is obtained from the bit error probability $P_{s}$ through

$$
P_{s}=\frac{2^{S F}-1}{2^{S F-1}} P_{b},
$$

then the probability that the sync word is badly estimated corresponds to the probability that at least one among the two independent symbols (in terms of observations) is badly estimated, which is expressed as

$$
P_{s w}=1-\left(1-P_{s}\right)^{2},
$$

where (3) or any of the BER expressions developed in the literature (see Table I) can be substituted in (4) and then in (5). Moreover, note that the term $\left(1-P_{s}\right)^{2}$ in (5) corresponds to the probability that both symbols are correctly estimated.

\section{B. Header Error Rate}

The header is composed of $N_{p l}=8$ symbols coded with Hamming code $\left(4, n_{H}=8\right)$ [10], therefore the header error rate $P_{h}$ can be deduced from (2) as

$$
P_{h}=1-\left(\left(1-P_{b}\right)^{8}+8 P_{b}\left(1-P_{b}\right)^{7}\right)^{S F} .
$$

\section{Frame Error Rate}

A frame is rejected when the sync word is badly estimated, or when the header or the payload is badly decoded. Thus, the general frame error probability $P_{f}$ can be expressed from (2), (5), and (6) using the inclusion-exclusion principle as

$$
P_{f}=1-\left(1-P_{s w}\right)\left(1-P_{h}\right)\left(1-P_{p l}\right) .
$$

It must be noticed in (7) that the FER is subject to the contributions of two coded sequences (the header and the payload), and one non-coded sequence (the sync word). We hereby develop an asymptotic analysis of the error probabilities $P_{s w}$, $P_{h}$, and $P_{p l}$ when the noise variance $\sigma^{2}$ reaches low values (or equivalently when the SNR is high).

\section{Asymptotic Performance}

In the following, we assume that $\sigma^{2}<<1$ such that $P_{b}<<$ 1. In this asymptotic condition, we analyze which contribution of the error rate $P_{s w}, P_{h}$, and $P_{p l}$ is dominant to the general FER $P_{f}$, i.e. which operation among the sync word estimation, the header decoding and the payload decoding is more likely to be erroneous. To this end, we rewrite $P_{p l}$ in (2) by using the Newton's general binomial theorem. For clarity purpose, we define $\beta=\frac{N_{p l} S F}{n_{H}}$, then $\beta \in \mathbb{Q}_{+}^{*}$. Then, since $P_{b}<<1$ we have

$$
\left(1-P_{b}\right)^{n_{H}}>n_{H} P_{b}\left(1-P_{b}\right)^{n_{H}-1}>0,
$$

allowing us to develop (2) as

$$
\begin{aligned}
P_{p l} & =1-\sum_{k=0}^{+\infty}\left(\begin{array}{l}
\beta \\
k
\end{array}\right)\left(1-P_{b}\right)^{n_{H}(\beta-k)}\left(n_{H} P_{b}\right)^{k}\left(1-P_{b}\right)^{\left(n_{H}-1\right) k} \\
& =1-\sum_{k=0}^{+\infty}\left(\begin{array}{l}
\beta \\
k
\end{array}\right)\left(n_{H} P_{b}\right)^{k}\left(1-P_{b}\right)^{n_{H} \beta-k}
\end{aligned}
$$

where $\left(\begin{array}{l}\beta \\ k\end{array}\right)$ is the generalized binomial coefficient, and $(1-$ $\left.P_{b}\right)^{n_{H} \beta-k}$ can also be rewritten by using the Newton's general binomial theorem: 


$$
\begin{aligned}
P_{p l}= & 1-\sum_{k=0}^{+\infty}\left(\begin{array}{l}
\beta \\
k
\end{array}\right)\left(n_{H} P_{b}\right)^{k} \sum_{m=0}^{+\infty}\left(\begin{array}{c}
n_{H} \beta-k \\
m
\end{array}\right)(-1)^{m} P_{b}^{m}, \\
= & -\sum_{k=1}^{+\infty}\left(\begin{array}{l}
\beta \\
k
\end{array}\right)\left(n_{H} P_{b}\right)^{k} \sum_{m=0}^{+\infty}\left(\begin{array}{c}
n_{H} \beta-k \\
m
\end{array}\right)(-1)^{m} P_{b}^{m}, \\
& -\sum_{m=1}^{+\infty}\left(\begin{array}{c}
n_{H} \beta \\
m
\end{array}\right)(-1)^{m} P_{b}^{m} .
\end{aligned}
$$

By keeping the terms of smallest degrees in (9), we obtain, after some developments:

$$
\begin{aligned}
P_{p l} & =\frac{\beta n_{H}\left(n_{H}-1\right)}{2} P_{b}^{2}+o\left(P_{b}^{2}\right) \\
& =\frac{N_{p l} S F\left(n_{H}-1\right)}{2} P_{b}^{2}+o\left(P_{b}^{2}\right) .
\end{aligned}
$$

Similarly, we have

$$
P_{h}=\frac{S F n_{H}\left(n_{H}-1\right)}{2} P_{b}^{2}+o\left(P_{b}^{2}\right) .
$$

Furthermore, the development of (4) yields

$$
P_{s w}=2 \frac{2^{S F}-1}{2^{S F-1}} P_{b}-\left(\frac{2^{S F}-1}{2^{S F-1}}\right)^{2} P_{b}^{2} .
$$

Then, by assuming that $N_{p l}>n_{H}$ (which is always true in practice), we obtain the asymptotic limits:

$$
\begin{gathered}
\lim _{P_{b} \rightarrow 0} \frac{P_{h}}{P_{p l}}=\frac{n_{H}}{N_{p l}}<1, \\
\lim _{P_{b} \rightarrow 0} \frac{P_{p l}}{P_{s w}}=\frac{N_{p l} S F\left(n_{H}-1\right) 2^{S F-1}}{4\left(2^{S F}-1\right)} P_{b}=0 .
\end{gathered}
$$

We deduce from (13) and (14) that, asymptotically we have $P_{h}<P_{p l}<P_{s w}$. Moreover, we deduce from (13) that $P_{h}$ and $P_{p l}$ have a similar behavior, and from (14) that $P_{s w}$ decreases slower than $P_{p l}$ (of order $O\left(P_{b}\right)$ for $P_{s w}$ versus order $O\left(P_{b}^{2}\right)$ for $\left.P_{p l}\right)$. This means that it exists an SNR value from which we have $P_{p l}<P_{s w}$. In practice, it signifies that in high SNR conditions (e.g. a device is close to the gateway), it is more likely to erroneously estimate the sync word and reject the frame, even when the header and the payload could be properly decoded. This behavior is verified through simulations, as presented in next Section.

\section{Simulations Results}

The simulations have been carried out using python 3.6 with scipy and gmpy 2 packages used for mathematical computing and precision improvement, respectively. The code can be found at https://github.com/b-com/fer_swer_LoRa. In fact, it has been stated in [5] that the computation of (3) leads to precision issues, due to the binomial coefficient $\left(\begin{array}{c}M-1 \\ k\end{array}\right)$ where $M$ is up to 4096 . In all simulations, we use similar parameters as [8], i.e. the Hamming code for the payload is $\left(k=4, n_{H}=7\right)$, and the payload length is $N_{p l}=32$. Note that it has been shown in [8] that the Monte-Carlo simulations

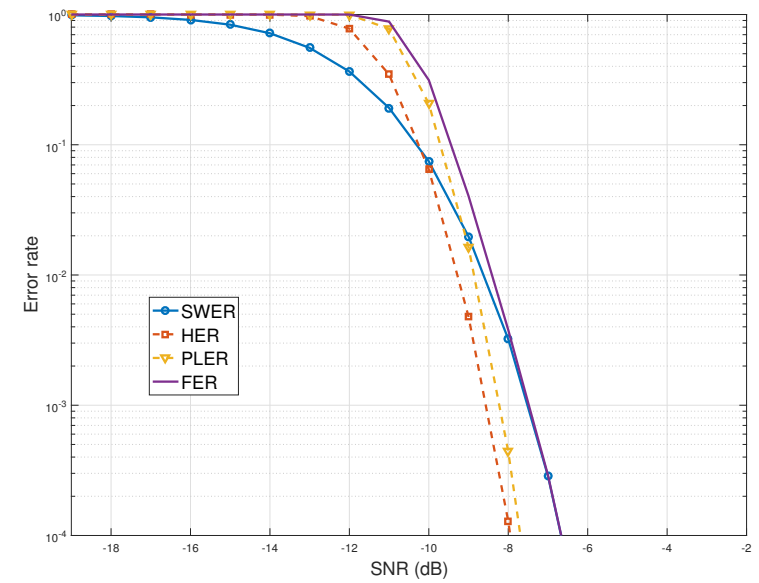

Fig. 3. Error rate versus SNR (dB), in AWGN channel and SF7.

match the the theoretical performance, therefore we can hereby focus on theoretical performance.

Fig. 3 shows the performance in terms of SWER, HER, PLER, and FER versus SNR (dB) for LoRa signal featuring SF7 over an AWGN channel. The theoretical results obtained from the asymptotic analysis are verified in Fig. 3. In fact, the HER and PLER have a similar behavior, in the sense that they have the same decreasing rate. Furthermore, SWER decreases slower than the HER and PLER, such that the FER is asymptotically equivalent to the SWER in the higher SNR range, and we observe that SWER $>$ PLER for $S N R>-9$ $\mathrm{dB}$.

Other series of simulations have been undertaken for a LoRa signal featuring SF7 over Rayleigh channel. The corresponding performance versus SNR $(\mathrm{dB})$ is shown in Fig. 4. Similar comments as previously can be drawn from Fig. 4, except that the error rates are higher than those in Fig. 3, due to the random nature of the considered channel. In that case, we find that SWER $>$ PLER for $S N R>5 \mathrm{~dB}$. Thus, we conclude from Figs. 3 and 4 that considering the BER performance only does not reflect all the source of errors and then underestimates the overall frame error rate.

\section{CONCLUSION}

In the LoRa protocol, a frame can be rejected if the frame header or the payload is badly decoded, or if the network identifier (sync word) is erroneously estimated. In this paper, we have proposed an improved expression of the frame error rate taking into account the three possible causes of rejection. Our analysis, corroborated with simulations, tend to prove that the impact of the SWER is not negligible in the whole SNR range and even tends to weaken the LoRa performance in the higher SNR range. To alleviate this problem, it may be possible to ignore the sync word at the PHY level and to let the MAC layer be responsible of frame rejection. 


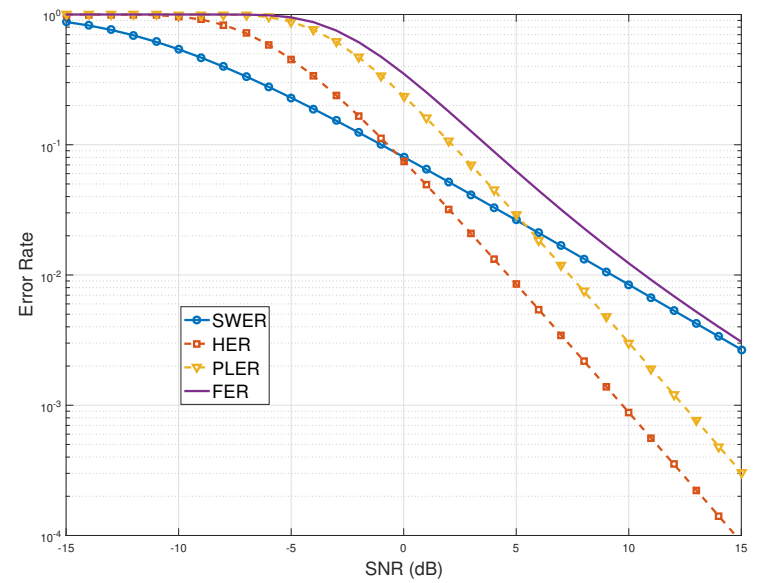

Fig. 4. Error rate versus SNR (dB), in Rayleigh channel and SF7.

\section{REFERENCES}

[1] T. Elshabrawy and J. Robert, "Closed-Form Approximation of LoRa Modulation BER Performance," IEEE Communications Letters, vol. 22, no. 9, pp. 1778 - 1781, September 2018.

[2] G. Ferré and A. Giremus, "LoRa Physical Layer Principle and Performance Analysis," in proc. of IEEE International Conference on Electronics, Circuits and Systems 2018 (ICECS'18). Bordeaux, France: IEEE, 12 2018, pp. 1 - 4.

[3] J. Proakis and M. Salehi, Digital Communications. McGraw-Hill, 2008.

[4] C. F. Dias, E. R. de Lima, and G. Fraidenraich, "Bit Error Rate ClosedForm Expressions for LoRa Systems under Nakagami and Rice Fading Channels," Sensors, vol. 19, no. 20, pp. 1 - 11, October 2019.

[5] V. Savaux and G. Ferré, "Simple Asymptotic BER Expressions for LoRaSystem over Rice and Rayleigh Channels," available in TechRxiv, vol. submitted in September, pp. 1-3, 2020.

[6] O. Afisiadis, M. Cotting, A. Burg, and A. Balatsoukas-Stimming, "On the Error Rate of the LoRa Modulation with Interference," IEEE Transactions on Wireless Communications, vol. 19, no. 2, pp. $1292-$ 1304, February 2020.

[7] M. J. Faber, K. M. van der Zwaag, W. G. V. dos Santos, H. R. d. O. Rocha, M. E. V. Segatto, and J. A. L. Silva, "A Theoretical and Experimental Evaluation on the Performance of LoRa Technology," IEEE Sensors Journal, vol. 20, no. 16, pp. 9480-9489, August 2020.

[8] O. Afisiadis, A. Burg, and A. Balatsoukas-Stimming, "Coded LoRa Frame Error Rate Analysis," ArXiv, no. arXiv:1911.10245v1, pp. 1 6, November 2019.

[9] M. Winkler, "Chirp signals for communications," IEEE WESCON Convention Record, p. 7, 1962.

[10] Semtech, "Datasheet SX1272/73," Semtech Corporation, Camarillo, CA, Tech. Rep., November 2015. 